\title{
Complete Derandomization of Identity Testing and Reconstruction of Read-Once Formulas
}

\author{
DANIEL MINAHAN and ILYA VOLKOVICH, University of Michigan
}

\begin{abstract}
In this article, we study the identity testing problem of arithmetic read-once formulas (ROFs) and some related models. An ROF is a formula (a circuit whose underlying graph is a tree) in which the operations are $\{+, \times\}$ and such that every input variable labels at most one leaf. We obtain the first polynomial-time deterministic identity testing algorithm that operates in the black-box setting for ROFs, as well as some other related models. As an application, we obtain the first polynomial-time deterministic reconstruction algorithm for such formulas. Our results are obtained by improving and extending the analysis of the algorithm of Shpilka and Yolkovich [51].
\end{abstract}

CCS Concepts: • Theory of computation $\rightarrow$ Algebraic complexity theory; Pseudorandomness and derandomization; Circuit complexity;

Additional Key Words and Phrases: Arithmetic circuit, arithmetic formula, derandomization, identity testing, circuit reconstruction

\section{ACM Reference format:}

Daniel Minahan and Ilya Volkovich. 2018. Complete Derandomization of Identity Testing and Reconstruction of Read-Once Formulas. ACM Trans. Comput. Theory 10, 3, Article 10 (May 2018), 11 pages.

https://doi.org/10.1145/3196836

\section{INTRODUCTION}

In this article, we study the problem of polynomial identity testing (PIT): given an arithmetic circuit $C$ over a field $\mathbb{F}$, with input variables $x_{1}, x_{2}, \ldots, x_{n}$, determine whether $C$ computes the identically zero polynomial. Given its connections to a wide range of problems, PIT is considered a central problem in algebraic complexity theory and algorithm design. Particular instances include perfect matchings in graphs [20, 39, 42], primality testing [2], IP = PSPACE [40, 47], the PCP theorem [9, $10]$, and many more. PIT is one of the few natural problems that have a simple efficient randomized algorithm $[18,46,54]$ but lack a deterministic one. Indeed, it has been a long-standing open question to come up with an efficient deterministic algorithm for this problem.

In this work, we consider the PIT problem in the black-box setting. In this setting, one is not given the full description of the circuit $C$ but is only allowed black-box (oracle) access to $C$. The problem of derandomizing identity testing in this setting reduces to that of finding for every $s$ an explicit set of points $\mathcal{H} \subseteq \mathbb{F}^{n}$ of size poly $(s)$ such that any nonzero circuit of size $s$ does not vanish on $\mathcal{H}$. We refer to such sets as hitting sets. Indeed, the randomized algorithm of DeMillo and Lipton [18], Schwartz [46], and Zippel [54] provides an exponential-size hitting set. Furthermore,

D. Minahan acknowledges the NSF for the support of his research.

Authors' address: D. Minahan and I. Volkovich, CSE Division, Bob and Betty Beyster Building 2260 Hayward, University of Michigan, Ann Arbor, MI 48109; emails: \{dminahan, ilyavol\}@umich.edu.

Permission to make digital or hard copies of all or part of this work for personal or classroom use is granted without fee provided that copies are not made or distributed for profit or commercial advantage and that copies bear this notice and the full citation on the first page. Copyrights for components of this work owned by others than ACM must be honored. Abstracting with credit is permitted. To copy otherwise, or republish, to post on servers or to redistribute to lists, requires prior specific permission and/or a fee. Request permissions from permissions@acm.org.

(C) 2018 ACM 1942-3454/2018/05-ART10 \$15.00

https://doi.org/10.1145/3196836 
applying standard probabilistic arguments, one can show the existence of "small" hitting sets. Yet coming up with an explicit hitting set is believed to be a very difficult task, as it would immediately imply explicit exponential lower bounds [1,29].

However, for several restricted classes of arithmetic circuits, efficient deterministic black-box PIT algorithms were found. For example, efficient black-box PIT algorithms were shown for depth-2 arithmetic circuits $[13,35,38]$ and depth-3 arithmetic circuits with bounded top fan-in (also known as $\Sigma \Pi \Sigma(k)$ circuits) [3, 11, 19, 32-34, 44, 45]. There has also been a lot of progress on PIT for restricted classes of depth-4 circuits [3, 7, 11, 12, 25, 31, 36, 37, 41, 43, 51]. Another body of research has been focused on PIT algorithms for bounded-read models-that is, classes of circuits where each variable appears some bounded number of times [4, 6, 7, 20-23, 26, 27, 51], with the simplest case being the read-once formulas (ROFs).

An ROF is an arithmetic formula (i.e., a tree) in which the operations are $\{+, \times\}$ and such that every input variable labels at most one leaf. These formulas can be thought of as the smallest formulas that depend on all of their variables and the simplest nontrivial subclass of multilinear formulas. Although ROFs form a very restricted model of computation, they have received a lot of attention in both the Boolean $[8,17,30]$ and algebraic $[14-16,28,50,51,53]$ worlds.

Although ROFs have a trivial polynomial (and, in fact, linear-time) white-box PIT algorithm, the first subexponential time $n^{O(\sqrt{n})}$ black-box PIT algorithm for ROFs was given in Shpilka and Volkovich [48]. Later on, in Shpilka and Volkovich [51], ${ }^{1}$ the result was improved to $n^{O(\log n)}$ via another algorithm. A different analysis for the latter algorithm, resulting in roughly the same runtime, was given in Anderson et al. [7]. Yet despite the rich body of work devoted to the problem, prior to our work no polynomial-time black-box PIT algorithm was known even for ROFs. In this article, we give the first black-box PIT algorithm for ROFs, and some related classes of formulas, thus achieving a complete derandomization of the PIT problem for these classes. For more information on PIT, we refer the reader to the survey of Shpilka and Yehudayoff [52].

It is important to point out that although PIT asks whether the resulting polynomial is identically zero as a formal sum of monomials, some nonidentically zero polynomials might evaluate to the zero function. For example, $x^{5}-x$ will always evaluate to zero over the field of five elements. For this reason, we will allow our algorithm to evaluate the polynomial on elements from a polynomially large extension field of $\mathbb{F}$. In Shpilka and Volkovich [51], it was shown that one cannot achieve polynomial-time black-box PIT algorithms if $|\mathbb{F}|=o(n / \log n)$.

\subsection{Our Results}

In this section, we describe and discuss our results. In fact, our results hold for the slightly richer class of preprocessed read-once formulas (PROFs). A preprocessed read-once formula is a ROF in which we are allowed to replace each variable $x_{i}$ with a univariate polynomial $T_{i}\left(x_{i}\right)$. A polynomial $P(\bar{x})$ is a preprocessed read-once polynomial (PROP) if it can be computed by a PROF. These PROPs also generalize the "sum-of-univariates" model. (See Section 3.2 for a formal definition.) We begin with our main result: polynomial-time black-box PIT algorithm for PROFs.

Theorem 1. Let $n, d \in \mathbb{N}$. There exists a deterministic algorithm that given black-box (oracle) access to a PROF $\Phi$ on $n$ variables and individual degrees (of the preprocessing) at most $d$, checks whether $\Phi \equiv 0$. The running time of the algorithm is polynomial in $n$ and $d$.

In Shpilka dn Volkovich [51], it was shown how to extend a PIT algorithm for a single PROF into a PIT algorithm for a sum of PROFs. By plugging in our main result, we obtain a black-box PIT algorithm for sums of PROFs.

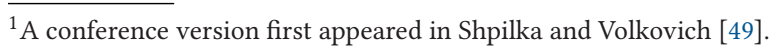


Theorem 2. Let $k, n, d \in \mathbb{N}$. There exists a deterministic algorithm that given black-box (oracle) access to $\Phi=\Phi_{1}+\cdots+\Phi_{k}$, where the $\Phi_{i}$ s are PROFs in $n$ variables, with individual degrees at most $d$, checks whether $\Phi \equiv 0$. The running time of the algorithm is $(n d){ }^{O(k)}$.

Observe that for a fixed $k \in \mathbb{N}$, the algorithm runs in polynomial time with respect to $n$ and $d$. Furthermore, observe that if $\mathcal{H}$ is a hitting set for a sum of two PROPs, then $\mathcal{H}$ is an interpolating set for a single PROP. In other words, the values of a single PROP $P$ on $\mathcal{H}$ contain enough information to uniquely identify $P$. Indeed, a consequence of Theorem 2 is an interpolating set of polynomial size for PROPs. However, in general, $\mathcal{H}$ does not provide us with an efficient algorithm to reconstruct a corresponding PROF.

In Bshouty et al. [16], a randomized polynomial-time reconstruction algorithm for ROFs was given. In Shpilka and Volkovich [50], the algorithm was extended to PROFs. Moreover, it was shown how to convert a black-box PIT algorithm into a reconstruction algorithm paying a polynomial overhead. Indeed, by plugging in the result of Shpilka and Volkovich [51], the first deterministic subexponential (and, in fact, quasipolynomial)-time reconstruction algorithm for PROFs was given. By plugging in our main result, we achieve a complete derandomization of the reconstruction algorithm by obtaining a deterministic polynomial-time reconstruction algorithm for PROFs.

THEOREM 3. There exists a deterministic algorithm that given black-box (oracle) access to a PROF $\Phi$, on $n$ variables and individual degrees at most $d$, reconstructs $\Phi$. Namely, the algorithm outputs a $P R O F \hat{\Phi}$ that computes the same polynomial. The running time of the algorithm is polynomial in $n$ and $d$.

\subsection{Organization}

The article is organized as follows. In Section 2, we give the basic definitions and notations. In Section 3, we formally introduce ROFs and their generalizations along with some structural properties, and in Section 3.3, we discuss the PIT algorithm of Shpilka and Volkovich [51]. In Section 3.4, we prove some additional properties of the algorithm, which is the main technical contribution of this work. Next, in Section 4, we give our main result, thus proving Theorem 1. We discuss the applications of our main result in Section 5, proving Theorems 2 and 3. We conclude the article with some open questions in Section 6.

\section{PRELIMINARIES}

For a positive integer $n$, let $[n]$ denote the set $\{1, \ldots, n\}$. We now give some definitions that apply to polynomials $P, Q \in \mathbb{F}\left[x_{1}, \ldots, x_{n}\right]$. For a polynomial $P$, a variable $x_{i}$, and $\alpha \in \mathbb{F}$, let $\left.P\right|_{x_{i}=\alpha}$ denote the polynomial that results upon setting $x_{i}=\alpha$. We say that $P$ depends on $x_{i}$ if and only if the degree of $x_{i}$ in $P$ is at least 1 . In other words, $P$ depends on $x_{i}$ if and only if $x_{i}$ appears when $P$ is written as a sum of monomials. We denote $\operatorname{var}(P) \triangleq\left\{i: P\right.$ depends on $\left.x_{i}\right\}$.

We say that $P$ is a homogeneous polynomial if all monomials in $P$ have the same total degree. For $i \in \mathbb{N}$, we define $H_{i}[P]$ as the homogeneous part of degree $i$ of $P$-that is, all monomials of total degree $i$ that appear in $P$. If $P$ does not have monomials of degree $i$, then $H_{i}[P] \equiv 0$. We say that $P$ and $Q$ are similar and denote $P \sim Q$ if there exists $\alpha \in \mathbb{F} \backslash\{0\}$ such that $\alpha \cdot P=Q$.

To actually calculate the complexity of our algorithm, we need to define a formal model of computation for polynomials.

Definition 2.1 (Arithmetic Formula). An arithmetic formula is a binary tree where each leaf is labeled with a variable $x_{i} \in\left\{x_{1}, \ldots, x_{n}\right\}$ and each internal node, called a gate, is labeled with an operation + or $\times$. Additionally, each leaf and node are labeled with some $(\alpha, \beta) \in \mathbb{F}^{2}$. The tree is 
evaluated by recursively calculating the values of the left subtree $P_{1}$ and the right subtree $P_{2}$ and then combining them by $\alpha\left(P_{1}\right.$ op $\left.P_{2}\right)+\beta$, where op is the operation at the top gate.

The efficiency of a formula over a set of formulas $C$ is measured by the number of gates in $C$. Thus, when we say polynomial time, we mean polynomial in the number of gates. Oftentimes, we will implicitly associate a class of formulas $C$ with the class of polynomials computed by these formulas.

We consider formulas in the black-box (or oracle) setting. In other words, the algorithm cannot directly look at the formula but rather is only allowed to use the formula to query the computed polynomials on points in $\mathbb{F}^{n}$. Hereafter, we assume that an evaluation query can be carried out in $O(1)$ time. In case $\mathbb{F}$ is small, we allow queries to the formula on a polynomially large extension field of $\mathbb{F}$.

\subsection{Generators and Hitting Sets}

Our black-box PIT algorithms use the notion of generators. In this section, we formally define this notion, describe a few of its useful properties, and give the connection to hitting sets. Intuitively, a generator $\mathcal{G}$ for a polynomial class $C$ is a function that stretches $t$ independent variables into $n \gg t$ dependent variables that can be plugged into any polynomial $P \in C$ without causing it to vanish.

Definition 2.2 (Hitting Set). Let $C$ be a class of polynomials in $\mathbb{F}\left[x_{1}, \ldots, x_{n}\right]$. A set $\mathcal{H}$ is called a hitting set for $C$ provided that $\forall P \in C$ with $P \not \equiv 0$, we have that $\left.P\right|_{\mathcal{H}} \not \equiv 0$.

This leads us to a basic algorithm for PIT.

Algorithm 1. Let $C$ be a class of polynomials in $\mathbb{F}\left[x_{1}, \ldots, x_{n}\right]$, and let $\mathcal{H}$ be a hitting set for $C$. Then there exists a deterministic PIT algorithm for $C$ that runs in time $O(|\mathcal{H}|)$.

The following generalization of the fundamental theorem of algebra provides hitting sets of exponential size for every polynomial. A proof can be found in Alon [5].

Lemma 2.3. Let $P \not \equiv 0 \in \mathbb{F}\left[x_{1}, \ldots, x_{n}\right]$, and suppose that the individual degree of any variable in $P$ is bounded by some $d \in \mathbb{N}$. Pick $S \subseteq \mathbb{F}$ with $|S|>d$. Then $\left.P\right|_{S^{n}} \not \equiv 0$.

Remark. The precondition of the lemma implies that $|\mathbb{F}|>d$. In case $\mathbb{F}$ is small, this assumption is met by choosing elements from an appropriately large extension field of $\mathbb{F}$.

A related notion is the notion of generators. Many hitting sets are constructed by means of generators.

Definition 2.4 (Generator). Let $C$ be a class of polynomials in $\mathbb{F}\left[x_{1}, \ldots, x_{n}\right]$. A polynomial map $G: \mathbb{F}^{t} \rightarrow \mathbb{F}^{n}$ is a generator for $C$ provided that $\forall P \not \equiv 0 \in C$ we have $P(G) \not \equiv 0$.

Intuitively, a generator $\mathcal{G}$ for $C$ is a polynomial mapping that has a hitting set for $C$ in its image. More specifically, Lemma 2.3 allows us to convert a generator into a hitting set by observing that a polynomial composed with a polynomial map results in another polynomial. Such composition typically reduces the number of variables on which the polynomial depends, but it may increase the total degree. Thus, since the size of the hitting set produced by Lemma 2.3 depends on both parameters, we want to find a generator that reduces the number of variables without drastically increasing its degree.

\section{READ-ONCE FORMULAS}

In this section, we discuss our computational model. We first consider the basic model of ROFs and cover some of its main properties. Then we introduce the model of the PROFs and give its corresponding properties. 


\subsection{ROFs and Read-Once Polynomials}

Most of the definitions that we give in this section are from Hancock and Hellerstein [28] and Shpilka and Volkovich [51] or some small variants. We start by formally defining the notions of an ROF and a read-once polynomial (ROP).

Definition 3.1 (Read-Once Formula). An read-once formula is an arithmetic formula where each variable appears at most once. A polynomial $P(\bar{x})$ is a ROP if it can be computed by an ROF.

Clearly, ROPs form a subclass of multilinear polynomials. In addition, note that the number of gates in a ROF is at most twice the number of variables. This means that our complexity scales with $n$, so we need only be concerned about how the runtime of our algorithm scales with respect to the number of variables. Thus, our ideal efficiency for an algorithm is $n^{O(1)}$. The next lemma also follows easily from the definition.

Lemma 3.2 (ROP Structural Lemma). Every ROP $P(\bar{x})$ that depends on at least two variables can be presented in exactly one of the following forms:

(1) $P(\bar{x})=P_{1}(\bar{x})+P_{2}(\bar{x})$

(2) $P(\bar{x})=P_{1}(\bar{x}) \cdot P_{2}(\bar{x})+c$,

where $P_{1}$ and $P_{2}$ are nonconstant, variable-disjoint ROPs and $c \in \mathbb{F}$ is a constant.

\subsection{Preprocessed Read-Once Polynomials}

In this section, we extend the model of ROFs by allowing a preprocessing step of the input variables. Whereas the basic model is read-once in its variables, the extended model can be considered as read-once in univariate polynomials. In addition, this model generalizes the "sum-of-univariates" model, which, in particular, contains the "sum-of-squares" model.

Definition 3.3. A preprocessing is a transformation $T(\bar{x}): \mathbb{F}^{n} \rightarrow \mathbb{F}^{n}$ of the form $T(\bar{x}) \triangleq$ $\left(T_{1}\left(x_{1}\right), T_{2}\left(x_{2}\right), \ldots, T_{n}\left(x_{n}\right)\right)$ such that each $T_{i}$ is a nonconstant univariate polynomial.

Notice that preprocessings do not affect the PIT problem in the white-box setting, as for every $n$-variate polynomial $P(\bar{y})$, it holds that $P(\bar{y}) \equiv 0$ if and only if $P(T(\bar{x})) \equiv 0$. We now give a formal definition and list some immediate properties.

Definition 3.4. A preprocessed arithmetic read-once formula (PROF) over a field $\mathbb{F}$ in the variables $\bar{x}=\left(x_{1}, \ldots, x_{n}\right)$ is a binary tree whose leaves are labeled with nonconstant univariate polynomials $T_{1}\left(x_{1}\right), T_{2}\left(x_{2}\right), \ldots, T_{n}\left(x_{n}\right)$ (all together forming a preprocessing) and whose internal nodes are labeled with the arithmetic operations $\{+, \times\}$. In addition, every node is labeled with a pair of field elements $(\alpha, \beta) \in \mathbb{F}^{2}$. Each $T_{i}$ can label at most one leaf. The computation is performed in the following way. A leaf labeled with the polynomial $T_{i}\left(x_{i}\right)$ and with $(\alpha, \beta)$ computes the polynomial $\alpha \cdot T_{i}\left(x_{i}\right)+\beta$. If a node $v$ is labeled with the operation op and with $(\alpha, \beta)$, and its children compute the polynomials $\Phi_{v_{1}}$ and $\Phi_{v_{2}}$, then the polynomial computed at $v$ is $\Phi_{v}=\alpha \cdot\left(\Phi_{v_{1}} o p \Phi_{v_{2}}\right)+\beta$.

A polynomial $P(\bar{x})$ is a preprocessed read-once polynomial if it can be computed by a PROF. A decomposition of a polynomial $P$ is a pair $Q(\bar{z}), T(\bar{x})$ such that $P(\bar{x})=Q(T(\bar{x}))$ when $Q$ is a $\mathrm{ROP}$ and $T$ is a preprocessing. An immediate consequence from the definition is that each PROP admits a decomposition. The following lemma is the PROPs analog of Lemma 3.2.

Lemma 3.5 (PROP Structural Lemma). Every PROP $P(\bar{x})$ with $|\operatorname{var}(P)| \geq 2$ can be presented in one of the following forms: 
(1) $P(\bar{x})=P_{1}(\bar{x})+P_{2}(\bar{x})$,

(2) $P(\bar{x})=P_{1}(\bar{x}) \cdot P_{2}(\bar{x})+c$,

where $P_{1}$ and $P_{2}$ are nonconstant, variable-disjoint PROPs and $c \in \mathbb{F}$ is a constant.

\subsection{The Algorithm of Shpilka and Volkovich}

In this article, we improve the complexity analysis of the PIT algorithm of Shpilka and Volkovich [51]. We begin by describing their algorithm. The heart of the algorithm is a construction of polynomial map $G_{n, t}$, which is shown to be a generator for PROPs for a certain range of parameters.

As in Shpilka and Volkovich [51], we fix a set $A=\left\{\alpha_{1}, \alpha_{2}, \ldots, \alpha_{n}\right\} \subseteq \mathbb{F}$ of $n$ distinct elements. It is also assumed that in case $\mathbb{F}$ is small, we have access to some extension field of $\mathbb{F}$ with more than $n$ elements. As was shown in Shpilka and Volkovich [51], this assumption is necessary to achieve a polynomial-time algorithm.

Definition 3.6 (The Generator of Shpilka and Volkovich [51]). Let $t \in \mathbb{N}$. For each $i \in[n]$, let $L_{i}(y)$ denote the $i$-th Lagrange interpolation polynomial. Formally, $L_{i}(y) \triangleq \frac{\prod_{j \neq i}\left(y-\alpha_{j}\right)}{\prod_{j \neq i}\left(\alpha_{i}-\alpha_{j}\right)}$. In other words, $L_{i}(y)$ is a degree $n-1$ polynomial satisfying $L_{i}\left(\alpha_{j}\right)=1$ when $j=i$ and $L_{i}\left(\alpha_{j}\right)=0$ when $j \neq i$. For each $i \in[n]$, let $G_{t}^{i}\left(y_{1}, \ldots, y_{t}, z_{1}, \ldots, z_{t}\right) \triangleq \sum_{k=1}^{t} L_{i}\left(y_{k}\right) \cdot z_{k}$. Finally, let $G_{n, t}\left(y_{1}, \ldots, y_{t}, z_{1}, \ldots, z_{t}\right) \triangleq\left(G_{t}^{1}\left(y_{1}, \ldots, z_{t}\right), \ldots, G_{t}^{n}\left(y_{1}, \ldots, z_{t}\right)\right)$.

$G_{n, t}$ can be seen as a sum of $t$ variable-disjoint copies of $G_{n, 1}$. This can be seen as the algebraic analogue of $t$-wise independent bits. The main part of the analysis of the algorithm is to establish that for every $n \in \mathbb{N}$, the map $G_{n, \log n}$ is a generator for PROPs on $n$ variables.

Lemma 3.7 ([51]). Let $P \in \mathbb{F}\left[x_{1}, \ldots, x_{n}\right]$ be a nonconstant PROP. Then $P\left(G_{n, \log n}\right)$ is nonconstant.

The intuition behind the proof is that a PROP can be written as either a sum or a product of two variable-disjoint polynomials (Lemma 3.5). Hence, (at least) one of these polynomials contains at most half of the variables. The map $G_{n, t}$ allows to "move" to a smaller polynomial by "shaving" a copy of $G_{n, 1}$. Finally, applying Lemma 2.3, one could show that if $G_{n, t}$ is a generator for a class of polynomials, then it can be converted into a relatively small hitting set for that same class.

LEMma 3.8. Let $P \in \mathbb{F}\left[x_{1}, \ldots, x_{n}\right]$ be a polynomial of degree $d$ such that $P\left(G_{n, t}\right) \not \equiv 0$ for some $t, d \in \mathbb{N}$. Then $P$ has a hitting set of size $(n d)^{O(t)}$.

Consequently, the result of Lemma 3.7 translates into a hitting set of size $(n d)^{O(\log n)}$ for PROPs.

We note that the generator of Shpilka and Volkovich [51] has been used as an ingredient in some subsequent PIT algorithms (e.g. [6, 7, 21, 24]).

\subsection{Our Technical Contribution}

In this section, we explore additional properties of the generator of Shpilka and Volkovich [51]. The main observation is a structural property of the generator when applied to a polynomial that depends only on a "small" subset of variables. This is the main technical contribution of our work.

Let $A=\left\{\alpha_{1}, \alpha_{2}, \ldots, \alpha_{n}\right\}$ be the set of elements that are used to define the generator.

Definition 3.9. For $I \subseteq[n]$, define $\Phi_{I}(y) \triangleq \prod_{i \in I}\left(y-\alpha_{i}\right)$. For notational convenience, $\Phi_{\emptyset}(y) \triangleq 1$.

To provide some intuition for the definition, we observe that for any $i \in[n]$, we have that $L_{i} \sim$ $\Phi_{[n] \backslash\{i\}}$.

Lemma 3.10. Let $P \in \mathbb{F}\left[x_{1}, \ldots, x_{n}\right]$ be a homogeneous polynomial of a total degree $d$, and let $\delta$ be an upper bound on the individual degrees of all variables $x_{i}$ in $P$. Then there exists a polynomial $\hat{P}(y)$ 
of degree at most $\delta \cdot|\operatorname{var}(P)|-d$ such that

$$
P\left(G_{n, 1}(y, z)\right)=z^{d} \cdot \Phi_{[n]}^{d-\delta}(y) \cdot \hat{P}(y) \cdot \Phi_{[n] \backslash \operatorname{var}(P)}^{\delta}(y) .
$$

In particular, there exists a polynomial $\hat{P}(y)$ of degree at most $d \cdot(|\operatorname{var}(P)|-1)$ such that

$$
P\left(G_{n, 1}(y, z)\right)=z^{d} \cdot \hat{P}(y) \cdot \Phi_{[n] \backslash \operatorname{var}(P)}^{d}(y) .
$$

Proof. Let $V \subseteq[n]$, and let $m(\bar{x})=\alpha \prod_{i \in V} x_{i}^{e_{i}}$ be a monomial subject to $\sum_{i \in V} e_{i}=d$ and $\forall i \in$ $V: 0 \leq e_{i} \leq \delta$.

$$
\begin{aligned}
& m\left(G_{n, 1}(y, z)\right)=\alpha z^{d} \cdot \prod_{i \in V} L_{i}^{e_{i}}(y)=\beta z^{d} \cdot \prod_{i \in V} \Phi_{[n] \backslash\{i\}}^{e_{i}}(y)=\beta z^{d} \cdot \Phi_{[n]}^{d}(y) / \prod_{i \in V}\left(y-\alpha_{i}\right)^{e_{i}} \\
& \quad=\beta z^{d} \cdot \Phi_{[n]}^{d-\delta}(y) \cdot \Phi_{V}^{\delta}(y) / \prod_{i \in V}\left(y-\alpha_{i}\right)^{e_{i}} \cdot \Phi_{[n] \backslash V}^{\delta}(y)=z^{d} \cdot \Phi_{[n]}^{d-\delta}(y) \cdot \beta \prod_{i \in V}\left(y-\alpha_{i}\right)^{\delta-e_{i}} \cdot \Phi_{[n] \backslash V}^{\delta}(y) .
\end{aligned}
$$

As was pointed out, $L_{i} \sim \Phi_{[n] \backslash\{i\}}$. Therefore, the purpose of $\beta \in \mathbb{F}$ is to "collect" all field constants when we move from $L_{i}$ to $\Phi_{[n] \backslash\{i\}}$. Next, take $\hat{m}(y)=\beta \prod_{i \in V}\left(y-\alpha_{i}\right)^{\delta-e_{i}}$ and observe that degree of $\hat{m}(y)$ is $\delta \cdot|V|-d$. By definition, the polynomial $P$ consists of a sum of such monomial $m(\bar{x})$ where $V=\operatorname{var}(P)$. Therefore, the first claim follows by a linearity argument. The second claim follows by observing that $d$ is an upper bound on the individual degrees of all variables $x_{i}$ in $P$, so we can set $\delta=d$.

\section{MAIN RESULT}

In this section, we prove our main result Theorem 1 . We begin by showing that $P\left(G_{n, 1}\right)$ hits sums of univariate polynomials. This proof is available in Shpilka and Volkovich [51], but we reproduce it here for completeness.

Lemma 4.1. Let $P \in \mathbb{F}\left[x_{1}, \ldots, x_{n}\right]$ be a nonconstant polynomial of the form $P=\sum_{i=1}^{n} T_{i}\left(x_{i}\right)$. Then $P\left(G_{n, 1}(y, z)\right)$ is nonconstant.

Proof. Pick $x_{i}$ such that $T_{i}\left(x_{i}\right)$ is nonconstant. Observe that $\left.P\left(G_{n, 1}\right)\right|_{y=\alpha_{i}}=T_{i}(z)+\sum_{j \neq i} T_{j}(0)$. This is a nonconstant polynomial, and so $P\left(G_{n, 1}\right)$ is nonconstant as well.

We now move to the proof our main result. We want to show that $G_{n, 1}$ is a generator for the set of PROPs. The idea is to proceed by induction using Lemma 3.5. Recall that for any $P \in \mathbb{F}\left[x_{1}, \ldots, x_{n}\right]$, and $i \in \mathbb{N}, H_{i}[P]$ denotes the homogeneous part of degree $i$ of $P$. Consequently, we can write $P=\sum_{i=0}^{d} H_{i}[P]$.

TheOrem 4.2. Let $P \in \mathbb{F}\left[x_{1}, \ldots, x_{n}\right]$ be a nonconstant PROP. Then $P\left(G_{n, 1}\right)$ is nonconstant.

Proof. Let $d$ denote the total degree of $P$. We induct on $m=|\operatorname{var}(P)|$. The base case where $m=1$ follows from Lemma 4.1. Now suppose that $m \geq 2$. By the PROP structural lemma (Lemma 3.5), we have two cases:

(1) $P=P_{1} \cdot P_{2}+c$. Note that $\left|\operatorname{var}\left(P_{1}\right)\right|,\left|\operatorname{var}\left(P_{2}\right)\right| \leq m-1$, so by the inductive hypothesis, $P_{1}\left(G_{n, 1}\right)$ and $P_{2}\left(G_{n, 1}\right)$ are nonconstant polynomials, and hence their product is nonconstant as well. Adding a constant does not affect this.

(2) $P=P_{1}+P_{2}$. For $j=1,2$, we can write $P_{j}=\sum_{i=0}^{d} P_{i, j}$, where $P_{i, j}=H_{i}\left[P_{j}\right]$. By Lemma 3.10, for each $0 \leq i \leq d$ and $j=1,2$, there exists a polynomial $\hat{P}_{i, j}(y)$ of degree at most $i \cdot\left(\left|\operatorname{var}\left(P_{i, j}\right)\right|-1\right)$ such that

$$
P_{j}\left(G_{n, 1}(y, z)\right)=\sum_{i=0}^{d} P_{i, j}\left(G_{n, 1}(y, z)\right)=\sum_{i=0}^{d} z^{i} \cdot \hat{P}_{i, j}(y) \cdot \Phi_{[n] \backslash \operatorname{var}\left(P_{i, j}\right)}^{i}(y)
$$


and hence

$$
P\left(G_{n, 1}(y, z)\right)=\sum_{i=0}^{d} z^{i} \cdot\left(\hat{P}_{i, 1}(y) \cdot \Phi_{[n] \backslash \operatorname{var}\left(P_{i, 1}\right)}^{i}(y)+\hat{P}_{i, 2}(y) \cdot \Phi_{[n] \backslash \operatorname{var}\left(P_{i, 2}\right)}^{i}(y)\right) .
$$

As before, by the inductive hypothesis, $P_{1}\left(G_{n, 1}\right)$ and $P_{2}\left(G_{n, 1}\right)$ are nonconstant polynomials. Therefore, there exist $1 \leq k \leq d$ such that

$$
z^{k} \cdot \hat{P}_{k, 1}(y) \cdot \Phi_{[n] \backslash \operatorname{var}\left(P_{k, 1}\right)}^{k}(y) \not \equiv 0
$$

and in particular $\hat{P}_{k, 1}(y) \not \equiv 0$. Let us denote $V_{j}=\operatorname{var}\left(P_{k, j}\right)$ and $W=[n] \backslash\left(V_{1} \cup V_{2}\right)$. Consider the expression that corresponds to the $z^{k}$ term in Equation (1):

$$
\hat{P}_{k, 1}(y) \cdot \Phi_{[n] \backslash \operatorname{var}\left(P_{k, 1}\right)}^{k}(y)+\hat{P}_{k, 2}(y) \cdot \Phi_{[n] \backslash \operatorname{var}\left(P_{k, 2}\right)}^{k}(y) .
$$

As $P_{k, 1}$ and $P_{k, 2}$ are variable disjoint, Equation (2) can be rewritten as

$$
\begin{aligned}
& \hat{P}_{k, 1}(y) \cdot \Phi_{V_{2} \cup W}^{k}(y)+\hat{P}_{k, 2}(y) \cdot \Phi_{V_{1} \cup W}^{k}(y) \\
& \quad=\Phi_{W}^{k}(y) \cdot\left(\hat{P}_{k, 1}(y) \cdot \Phi_{V_{2}}^{k}(y)+\hat{P}_{k, 2}(y) \cdot \Phi_{V_{1}}^{k}(y)\right) .
\end{aligned}
$$

The last equality follows from the properties of $\Phi$ (see Definition 3.9).

We claim that the obtained expression is nonconstant. To this end, it is sufficient to show that $\hat{P}_{k, 1}(y) \cdot \Phi_{V_{2}}^{k}(y)+\hat{P}_{k, 2}(y) \cdot \Phi_{V_{1}}^{k}(y) \not \equiv 0$. Assume the contrary. We obtain that $\hat{P}_{k, 1}(y) \cdot \Phi_{V_{2}}^{k}(y)=-\hat{P}_{k, 2}(y) \cdot \Phi_{V_{1}}^{k}(y)$. As $V_{1}$ and $V_{2}$ are disjoint sets, $\Phi_{V_{1}}(y)$ and $\Phi_{V_{2}}(y)$ have no common roots. Therefore, it must be the case that $\Phi_{V_{1}}^{k}$ divides $\hat{P}_{k, 1}$. As $\hat{P}_{k, 1} \not \equiv 0$, we get that

$$
\operatorname{deg}\left(\hat{P}_{k, 1}\right) \geq \operatorname{deg}\left(\Phi_{V_{1}}^{k}\right)=k\left|V_{1}\right|,
$$

whereas by Lemma 3.10, $\hat{P}_{k, 1}(y)$ is a polynomial of degree at most $k \cdot\left(\left|V_{1}\right|-1\right)$. Consequently, the coefficient of $z^{k}$ in $P\left(G_{n, 1}(y, z)\right)$ is nonconstant and the claim follows.

Theorem 1 follows by combining Theorem 4.2 with Lemma 3.8 .

\section{APPLICATIONS}

In this section, we show two applications for our main result, proving Theorems 2 and 3 . The first application is testing whether several PROPs sum up to the zero polynomial. To this end, we require the following result, which shows that a generator for the class of PROPs can be extended to yield a generator for the class of sums of PROPs.

LEMma 5.1 ([51]). Let $\mathcal{G}_{n}$ be a generator for PROPs on $n$ variables. Then for any $k \in \mathbb{N}, \mathcal{G}_{n}+G_{n, 3 k}$ is a generator for sums of $k$ PROPs on $n$ variables.

Remark. As both $\mathcal{G}_{n}$ and $G_{n, 3 k}$ represent polynomial maps with the same output length, the sum $\mathcal{G}+G_{n, 3 k}$ should be interpreted as a componentwise sum, where we implicitly assume that the variables of $\mathcal{G}_{n}$ and $G_{n, 3 k}$ have been relabeled to be disjoint. The next corollary follows by combining Lemma 5.1 with Theorem 4.2 and the properties of $G_{n, t}$ (see Definition 3.6).

COROLlary 5.2. For any $k, n \in \mathbb{N}$, the map $G_{n, 3 k+1}$ is a generator for sums of $k$ PROPs on $n$ variables.

Theorem 2 follows by applying Lemma 3.8. Observe that if $\mathcal{H}$ is a hitting set for a sum of two PROPs, then $\mathcal{H}$ is an interpolating set for a single PROP. In other words, the values of a single PROP $P$ on $\mathcal{H}$ contain enough information to uniquely identify $P$. Indeed, a consequence of Theorem 2 is an interpolating set of polynomial size for PROPs. However, in general, $\mathcal{H}$ does not provide us 
with an efficient algorithm to reconstruct a corresponding PROF. In Shpilka and Volkovich [50], it was shown how to turn a hitting set for PROPs into a reconstruction algorithm with a polynomial overhead.

Lemma 5.3 ([50]). Let $n, d \in \mathbb{N}$. There exists a deterministic algorithm that given a hitting set $\mathcal{H}_{n, d}$ for PROPs on $n$ variable and degree at most $d$, and black-box (oracle) access to a PROP P as earlier, outputs a PROF $\Phi$ that computes $P$ in time polynomial in $n, d$, and $\left|\mathcal{H}_{n, d}\right|$.

Combining the lemma with Theorem 1 results in Theorem 3. Indeed, the original reconstruction algorithm of Shpilka and Volkovich [50] used the quasi-polynomial-size hitting set of Shpilka and Volkovich [51].

\section{CONCLUSIONS AND OPEN QUESTIONS}

In this article, we present the first polynomial-time black-box identity testing and reconstruction algorithms for ROFs, which form a subclass of multilinear formulas. In Anderson et al. [7], quasipolynomial-time and polynomial-time PIT algorithms were given for multilinear read- $k$ formulas in the black-box and the white-box settings, respectively, for constant values of $k$. At a high level, both algorithms go by alternating the following two steps:

- Step 1: Reduce PIT of a read- $(k+1)$ formula to PIT of the sum of two read- $k$ formulas.

- Step 2: Reduce PIT of a sum of two read- $k$ formulas to PIT of a (single) read- $k$ formula.

Although Step 2 introduces an overhead of (roughly) $n^{k^{O(k)}}$ in both settings, the gap in the final complexity results from the overhead introduced by Step 1. Indeed, in the white-box setting, the overhead is $\operatorname{poly}(n, k)$, whereas in the black-box setting, the overhead is $n^{O(\log n)}$. Moreover, for $k=0$, the analysis of Step 2 can be seen as a different analysis of the black-box PIT algorithm for ROFs of Shpilka and Volkovich [51], resulting in roughly the same runtime. It is our hope that the ideas presented in this article could be extended further to improve the analysis of the black-box PIT algorithms of Anderson et al. [7] and perhaps lead to new PIT algorithms.

Some open questions are the following. Can one obtain a polynomial-time black-box PIT algorithm for a multilinear read- $k$ formula with a constant $k$ ? What about $k=2$ (i.e., multilinear readtwice formulas)? Even more specifically, can one show a black-box reduction from a PIT instance of a multilinear read-twice formula to polynomially many PIT instances of sums of constantly many ROFs, introducing only a polynomial overhead?

\section{ACKNOWLEDGMENTS}

The authors would like to thank the anonymous referees for useful comments that improved the presentation of the results.

\section{REFERENCES}

[1] M. Agrawal. 2005. Proving lower bounds via pseudo-random generators. In FSTTCS 2005: Foundations of Software Technology and Theoretical Computer Science. Lecture Notes in Computer Science, Vol. 3821. Springer, 92-105.

[2] M. Agrawal, N. Kayal, and N. Saxena. 2004. PRIMES is in P. Annals of Mathematics 160, 2, 781-793.

[3] M. Agrawal, C. Saha, R. Saptharishi, and N. Saxena. 2012. Jacobian hits circuits: Hitting-sets, lower bounds for depthD occur-k formulas and depth-3 transcendence degree-k circuits. In Proceedings of the 44th Annual ACM Symposium on Theory of Computing (STOC'12). 599-614.

[4] M. Agrawal, C. Saha, and N. Saxena. 2013. Quasi-polynomial hitting-set for set-depth-Delt a formulas. In Proceedings of the 45th Annual ACM Symposium on Theory of Computing (STOC'13). 321-330.

[5] N. Alon. 1999. Combinatorial nullstellensatz. Combinatorics, Probability and Computing 8, 7-29.

[6] M. Anderson, M. A. Forbes, R. Saptharishi, A. Shpilka, and B. L. Volk. 2016. Identity testing and lower bounds for read- $k$ oblivious algebraic branching programs. In Proceedings of the 31st Conference on Computational Complexity (CCC'16). 30:1-30:25. https://doi.org/10.4230/LIPIcs.CCC.2016.30 
[7] M. Anderson, D. van Melkebeek, and I. Volkovich. 2015. Derandomizing polynomial identity testing for multilinear constant-read formulae. Computational Complexity 24, 4, 695-776.

[8] D. Angluin, L. Hellerstein, and M. Karpinski. 1993. Learning read-once formulas with queries. Journal of the ACM 40, 1, 185-210.

[9] S. Arora, C. Lund, R. Motwani, M. Sudan, and M. Szegedy. 1998. Proof verification and the hardness of approximation problems. Fournal of the ACM 45, 3, 501-555.

[10] S. Arora and S. Safra. 1998. Probabilistic checking of proofs: A new characterization of NP. fournal of the ACM 45, 1 , $70-122$.

[11] V. Arvind and P. Mukhopadhyay. 2010. The monomial ideal membership problem and polynomial identity testing. Information and Computation 208, 4, 351-363.

[12] M. Beecken, J. Mittmann, and N. Saxena. 2011. Algebraic independence and blackbox identity testing. In Proceedings of the 38th International Colloquium on Automata, Languages, and Programming (ICALP'11). 137-148.

[13] M. Ben-Or and P. Tiwari. 1988. A deterministic algorithm for sparse multivariate polynominal interpolation. In Proceedings of the 20th Annual ACM Symposium on Theory of Computing (STOC'88). 301-309.

[14] D. Bshouty and N. H. Bshouty. 1998. On interpolating arithmetic read-once formulas with exponentiation. Fournal of Computer and System Sciences 56, 1, 112-124.

[15] N. H. Bshouty and R. Cleve. 1998. Interpolating arithmetic read-once formulas in parallel. SIAM fournal on Computing 27, 2, 401-413.

[16] N. H. Bshouty, T. R. Hancock, and L. Hellerstein. 1995. Learning arithmetic read-once formulas. SIAM fournal on Computing 24, 4, 706-735.

[17] N. H. Bshouty, T. R. Hancock, and L. Hellerstein. 1995. Learning Boolean read-once formulas with arbitrary symmetric and constant fan-in gates. Journal of Computer and System Sciences 50, 521-542.

[18] R. A. DeMillo and R. J. Lipton. 1978. A probabilistic remark on algebraic program testing. Information Processing Letters 7, 4, 193-195.

[19] Z. Dvir and A. Shpilka. 2006. Locally decodable codes with 2 queries and polynomial identity testing for depth 3 circuits. SIAM Journal on Computing 36, 5, 1404-1434.

[20] S. A. Fenner, R. Gurjar, and T. Thierauf. 2016. Bipartite perfect matching is in quasi-NC. In Proceedings of the 48th Annual ACM SIGACT Symposium on Theory of Computing (STOC'16). 754-763. https://doi.org/10.1145/2897518.2897564

[21] M. A. Forbes, R. Saptharishi, and A. Shpilka. 2014. Pseudorandomness for multilinear read-once algebraic branching programs, in any order. In Proceedings of the 46th Annual ACM Symposium on Theory of Computing (STOC'14). 867875. https://doi.org/10.1145/2591796.2591816 Full version at https://eccc.weizmann.ac.il/report/2013/132.

[22] M. A. Forbes and A. Shpilka. 2013. Explicit Noether normalization for simultaneous conjugation via polynomial identity testing. In Approximation, Randomization, and Combinatorial Optimization, Algorithms and Techniques. Lecture Notes in Computer Science, Vol. 8096. Springer, 527-542.

[23] M. A. Forbes and A. Shpilka. 2013. Quasipolynomial-time identity testing of non-commutative and read-once oblivious algebraic branching programs. In Proceedings of the 54th Annual IEEE Symposium on Foundations of Computer Science (FOCS'13). 243-252. https://doi.org/10.1109/FOCS.2013.34

[24] M. A. Forbes, A. Shpilka, I. Tzameret, and A. Wigderson. 2016. Proof complexity lower bounds from algebraic circuit complexity. In Proceedings of the 31st Conference on Computational Complexity (CCC'16). 32:1-32:17.

[25] A. Gupta. 2014. Algebraic geometric techniques for depth-4 PIT and Sylvester-Gallai conjectures for varieties. Electronic Colloquium on Computational Complexity 21, 130. https://eccc.weizmann.ac.il/report/2014/130

[26] R. Gurjar, A. Korwar, and N. Saxena. 2016. Identity testing for constant-width, and commutative, read-once oblivious ABPs. In Proceedings of the 31st Conference on Computational Complexity (CCC'16). 29:1-29:16. https://doi.org/10. 4230/LIPIcs.CCC.2016.29

[27] R. Gurjar, A. Korwar, N. Saxena, and N. Thierauf. 2015. Deterministic identity testing for sum of read-once oblivious arithmetic branching programs. In Proceedings of the 30th Conference on Computational Complexity (CCC'15). 323-346. https://doi.org/10.4230/LIPIcs.CCC.2015.323

[28] T. R. Hancock and L. Hellerstein.1991. Learning read-once formulas over fields and extended bases. In Proceedings of the 4th Annual Workshop on Computational Learning Theory (COLT'91). 326-336.

[29] J. Heintz and C. P. Schnorr. 1980. Testing polynomials which are easy to compute (extended abstract). In Proceedings of the 12th Annual ACM Symposium on Theory of Computing (STOC'80). 262-272.

[30] M. Karchmer, N. Linial, I. Newman, M. E. Saks, and A. Wigderson. 1993. Combinatorial characterization of read-once formulae. Discrete Mathematics 114, 1-3, 275-282.

[31] Z. S. Karnin, P. Mukhopadhyay, A. Shpilka, and I. Volkovich. 2013. Deterministic identity testing of depth 4 multilinear circuits with bounded top fan-in. SIAM fournal on Computing 42, 6, 2114-2131.

[32] Z. S. Karnin and A. Shpilka. 2011. Black box polynomial identity testing of generalized depth-3 arithmetic circuits with bounded top fan-in. Combinatorica 31, 3, 333-364. https://doi.org/10.1007/s00493-011-2537-3 
[33] N. Kayal and S. Saraf. 2009. Blackbox polynomial identity testing for depth 3 circuits. In Proceedings of the 50th Annual IEEE Symposium on Foundations of Computer Science (FOCS'09). 198-207. https://eccc.weizmann.ac.il/report/2009/032

[34] N. Kayal and N. Saxena. 2007. Polynomial identity testing for depth 3 circuits. Computational Complexity 16, 2, 115138.

[35] A. Klivans and D. Spielman. 2001. Randomness efficient identity testing of multivariate polynomials. In Proceedings of the 33rd Annual ACM Symposium on Theory of Computing (STOC'01). 216-223.

[36] M. Kumar and S. Saraf. 2016. Arithmetic circuits with locally low algebraic rank. In Proceedings of the 31st Conference on Computational Complexity (CCC'16). 34:1-34:27. https://doi.org/10.4230/LIPIcs.CCC.2016.34

[37] M. Kumar and S. Saraf. 2016. Sums of products of polynomials in few variables: Lower bounds and polynomial identity testing. In Proceedings of the 31st Conference on Computational Complexity (CCC'16). 35:1-35:29. https://doi.org/10. 4230/LIPIcs.CCC.2016.35

[38] R. J. Lipton and N. K. Vishnoi. 2003. Deterministic identity testing for multivariate polynomials. In Proceedings of the 14th Annual ACM-SIAM Symposium on Discrete Algorithms (SODA'03). 756-760.

[39] L. Lovasz. 1979. On determinants, matchings, and random algorithms. In Fundamentals of Computing Theory, L. Budach (Ed.). Akademia-Verlag.

[40] C. Lund, L. Fortnow, H. Karloff, and N. Nisan. 1992. Algebraic methods for interactive proof systems. Fournal of the ACM 39, 4, 859-868.

[41] P. Mukhopadhyay. 2016. Depth-4 identity testing and Noether's normalization lemma. In Proceedings of the 11th International Computer Science Symposium on Computer Science-Theory and Applications-Volume 9691. (CSR'16). 309-323.

[42] K. Mulmuley, U. Vazirani, and V. Vazirani. 1987. Matching is as easy as matrix inversion. Combinatorica 7, 1, 105-113.

[43] N. Saxena. 2008. Diagonal circuit identity testing and lower bounds. In Proceedings of the 35th International Colloquium on Automata, Languages, and Programming. 60-71. https://eccc.weizmann.ac.il/eccc-reports/2007/TR07-124/index. html.

[44] N. Saxena and C. Seshadhri. 2010. From Sylvester-Gallai configurations to rank bounds: Improved black-box identity test for deph-3 circuits. In Proceedings of the 51st Annual IEEE Symposium on Foundations of Computer Science (FOCS'10). 21-30.

[45] N. Saxena and C. Seshadhri. 2011. An almost optimal rank bound for depth-3 identities. SIAM fournal on Computing $40,1,200-224$.

[46] J. T. Schwartz. 1980. Fast probabilistic algorithms for verification of polynomial identities. fournal of the ACM 27, 4, 701-717.

[47] A. Shamir. 1990. IP=PSPACE. In Proceedings of the 31st Annual Symposium on Foundations of Computer Science (FOCS'90). 11-15.

[48] A. Shpilka and I. Volkovich. 2008. Read-once polynomial identity testing. In Proceedings of the 40th Annual ACM Symposium on Theory of Computing (STOC'08). 507-516. https://doi.org/10.1145/1374376.1374448

[49] A. Shpilka and I. Volkovich. 2009. Improved polynomial identity testing for read-once formulas. In Proceedings of the 12th International Workshop and 13th International Workshop on Approximation, Randomization, and Combinatorial Optimization: Algorithms and Techniques (APPROX'09/RANDOM'09). 700-713. https://eccc.weizmann.ac.il/report/ $2010 / 011$.

[50] A. Shpilka and I. Volkovich. 2014. On reconstruction and testing of read-once formulas. Theory of Computing 10, 465-514.

[51] A. Shpilka and I. Volkovich. 2015. Read-once polynomial identity testing. Computational Complexity 24, 3, 477-532.

[52] A. Shpilka and A. Yehudayoff. 2010. Arithmetic circuits: A survey of recent results and open questions. Foundations and Trends in Theoretical Computer Science 5, 3-4, 207-388.

[53] I. Volkovich. 2016. Characterizing arithmetic read-once formulae. ACM Transactions on Computation Theory 8, 1, 2. https://doi.org/10.1145/2858783

[54] R. Zippel. 1979. Probabilistic algorithms for sparse polynomials. In Proceedings of the International Symposium on Symbolic and Algebraic Computation. 216-226.

Received July 2017; revised January 2018; accepted January 2018 